\title{
Analysis of the X-ray spectrum of the hot bubble of $\mathrm{BD}+30^{\circ} 3639$
}

\author{
Detlef Schönberner, Ralf Jacob, René Heller $†$ and Matthias Steffen \\ Leibniz-Institut für Astrophysik (AIP), An der Sternwarte 16, 14482 Potsdam, Germany \\ email: deschoenberner@aip.de
}

\begin{abstract}
We developed a model for wind-blown bubbles with temperature and density profiles based on self-similar solutions including thermal conduction. We constructed also heatconduction bubbles with chemical discontinuities. The X-ray emission is computed using the well-documented CHIANTI code (v6.0.1). These bubble models are used to (re)analyse the high-resolution X-ray spectrum of the hot bubble of $\mathrm{BD}+30^{\circ} 3639$, and they appeared to be much superior to constant temperature approaches.

We found for the X-ray emission of $\mathrm{BD}+30^{\circ} 3639$ that temperature-sensitive and abundancesensitive line ratios computed on the basis of heat-conducting wind-blown bubbles and with abundances as found in the stellar photosphere/wind can only be reconciled with the observations if the hot bubble of $\mathrm{BD}+30^{\circ} 3639$ is chemically stratified, i.e. if it contains also a small mass fraction $(\simeq 3 \%)$ of hydrogen-rich matter immediately behind the conduction front. Neon appears to be strongly enriched, with a mass fraction of at least about 0.06 .
\end{abstract}

Keywords. conduction, planetary nebulae: individual: $\mathrm{BD}+30^{\circ} 3639$, stars: abundances, X-rays: stars

\section{Introduction}

The cavities of elliptical/roundish planetary nebulae (PNe) are not empty but instead filled-up with hot gas originating from the shock-heated fast stellar wind. Space-born X-ray telescopes (ROSAT, XMM-Newton, Chandra) revealed that these wind-blown "bubbles" are the source of rather soft X-ray emission, with typical plasma temperatures between 1 and $3 \mathrm{MK}$ (see Kastner et al. 2012). The low plasma temperature observed is substantially below the postshock temperature of about $10 \mathrm{MK}$ expected from the high observed velocities of the stellar wind. Possible "cooling" processes are either heat conduction in the absence of magnetic fields (Soker 1994; Steffen et al. 2008) and/or dynamical mixing of matter across the bubble/nebula interface (Toalá \& Arthur 2016). The latest 2D simulations by Toalá \& Arthur (2016) suggest that heat conduction is indispensable for achieving the observed high X-ray emission measures.

The case of $\mathrm{BD}+30^{\circ} 3639$ is special in three aspects: i) A hydrogen-poor wind interacts with a nebular shell of normal, hydrogen-rich composition; ii) it is the brightest PN X-ray source, allowing to take high-resolution spectra (Yu et al. 2009); iii) it is a young, still unevolved nebula, obviously shortly after the conversion to a hydrogen-poor central star has taken place. A detailed analysis of the X-ray spectrum with respect to elemental abundance ratios and the existence of a possible abundance discontinuity within the bubble produced by heat conduction is thus very rewarding.

All the existing spectral analyses of this nebula are based on simple plasma models with constant densities and temperatures. In their analysis of the bubble of $\mathrm{BD}+30^{\circ} 3639$, Yu et al. (2009) and Nordon et al. (2009) needed a two-temperature plasma (1.9 and 3.0 MK) to account for the emission from species with different degree of ionisation. The derived chemical abundances appeared to be rather unusual: very high ratios of $\mathrm{C} / \mathrm{O}$ and $\mathrm{Ne} / \mathrm{O}$, exceeding by far the corresponding solar ratios.

$\dagger$ Present address: Max-Planck-Institut für Sonnensystemforschung, Justus-von-Liebig-Weg 3, 37077 Göttingen, Germany 

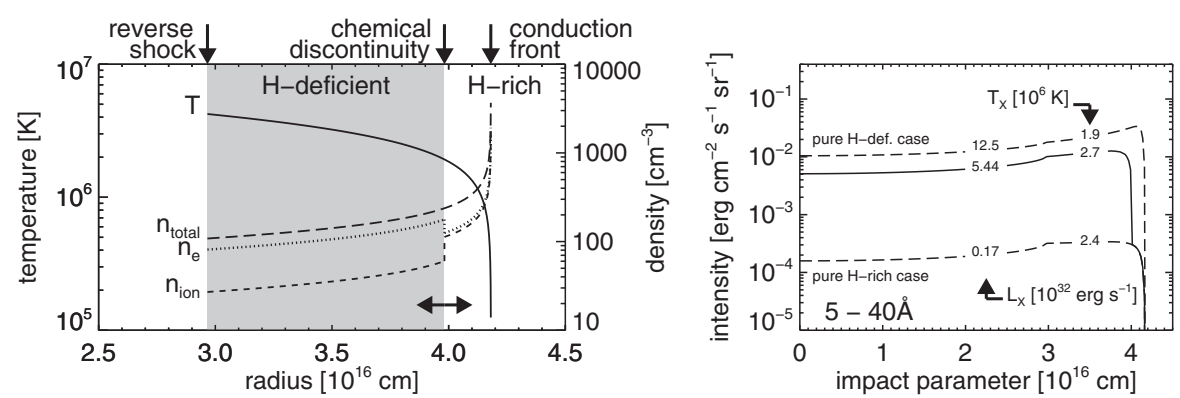

Figure 1. Left: physical structure of a ZP96 bubble of age $=500 \mathrm{yr}$ with a stratified composition such that $\omega \equiv M_{\mathrm{PN}} /\left(M_{\mathrm{WR}}+M_{\mathrm{PN}}\right)=0.03$. Shown are radial runs of electron, ion, and total particle densities, and the electron temperature. The reverse wind shock, the chemical discontinuity, and the conduction front are marked by vertical arrows. The condition of constant pressure across the bubble leads to the density jumps at the chemical boundary at $r \simeq 4 \times 10^{16} \mathrm{~cm}$, or 2 MK. Right: the model's X-ray surface brightness integrated over $5-40 \AA$. The dashed curves serve for comparisons with the homogeneous WR (H-poor) and PN (H-rich) cases only. Remarkably is the high emission of the WR matter which is exclusively residing in the hotter bubble regions. Thus, $T_{\mathrm{X}}$ is increased from 1.9 to $2.7 \mathrm{MK}$ for the $\omega=0.03$ case shown here, while the $\mathrm{X}$-ray luminosity is more than halved because of the low PN-matter emissivity.

From the physical point of view, such a low-temperature plasma is fully inconsistent with its outer and inner boundary conditions, viz. the nebular shell and the fast stellar wind. In the following we report briefly on our new approach to analyse the X-ray emission from hot bubbles by means of a physically more sound model. Details will be published in a forthcoming journal paper.

\section{Bubble models}

Our newly developed analysis tool is based on self-similar solutions for wind-blown bubbles formed by the interaction of two spherical winds with different densities and velocities, as developed by Zhekov \& Perinotto $(1996,1998)$. These so-called ZP96-bubbles contain heat conduction across the bubble from the reverse wind shock to the contact discontinuity/conduction front. The general properties of these bubbles can be characterised as follows:

Inner boundary condition. Power-law representation of the fast stellar wind from an evolving $0.595 \mathrm{M}_{\odot}$ central star, time-dependent mass-loss rate and velocity, adapted for $\mathrm{BD}+30^{\circ} 3639$ according to Sandin et al. (2016).

Outer boundary condition. Constant slow wind with explored parameter ranges $10^{-7} \ldots 10^{-4}$ $\mathrm{M}_{\odot} \mathrm{yr}^{-1}$ and $10 \ldots 40 \mathrm{~km} \mathrm{~s}^{-1}$.

Chemical composition. Each bubble has either a hydrogen-deficient ("WR"; Marcolino et al. 2007) or hydrogen-rich ("PN") chemical composition, with appropriate conduction coefficients. Also, chemically inhomogeneous bubbles are constructed to allow for "evaporated" PN matter behind the conduction front. The WR composition is extremely helium-, carbon-, and oxygenrich: He:C:O $=0.43: 0.51: 0.06$ by mass (Marcolino et al. 2007).

$X$-ray emission. The X-ray spectrum is computed by means of the well-documented CHIANTI code, v6.0.1 (Dere et al. 2009).

Altogether, about 1000 bubbles have been generated, spanning ages from 200 to 1000 years, corresponding characteristic X-ray temperatures, $T_{\mathrm{X}}$ (Eq. 17 in Steffen et al. 2008), between 1.1 and $3.9 \mathrm{MK}$ (WR) or between 1.1 and 6.8 MK (PN). WR-bubbles with ages of 400-500 yr have sizes and X-ray luminosities which correspond well to the respective values observed for $\mathrm{BD}+30^{\circ} 3639$. The structure of a bubble with a chemical discontinuity is displayed in Fig. 1 . 

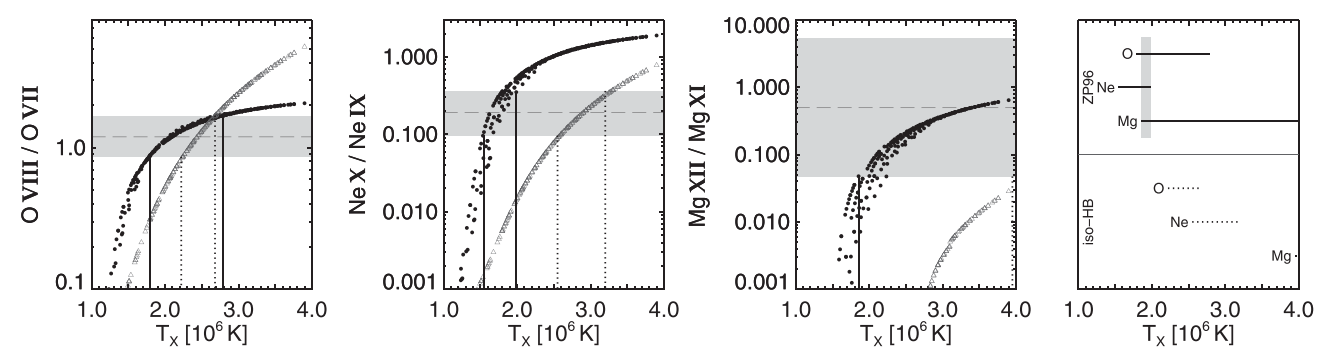

Figure 2. Line ratios for two ionisation stages of oxygen (left), neon (next to left), and magnesium (next to right) vs. $T_{\mathrm{X}}$ as predicted by our ZP96 bubble models with hydrogen-deficient WR composition (dots) and constant-temperature bubbles (triangles) with the same chemical composition. The horizontal long-dashed lines give the measured values for $\mathrm{BD}+30^{\circ} 3639$, the shaded regions indicate their uncertainties. The right panel summarises the $T_{\mathrm{X}}$ determinations from the various line ratios and for the ZP96-bubbles and the constant-temperature approach.
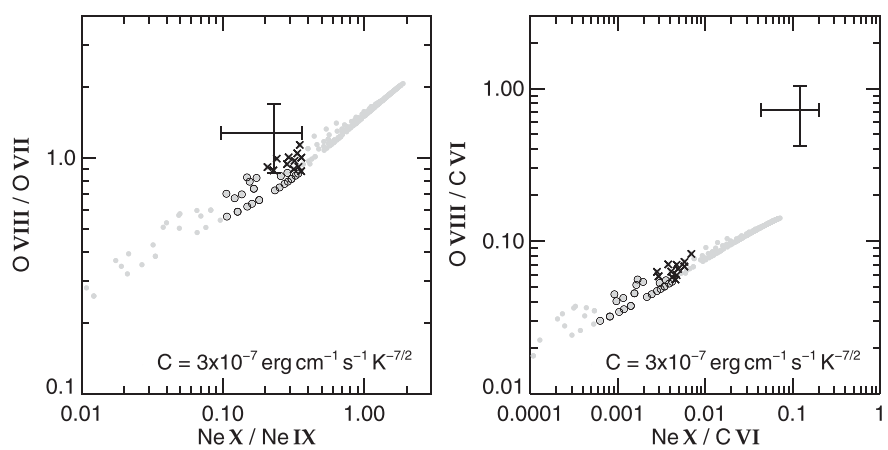

Figure 3. Temperature sensitive line ratios of oxygen and neon (left) and abundance sensitive line ratios (right) as predicted by bubbles with homogeneous WR composition and observed values for $\mathrm{BD}+30^{\circ} 3639$ (big error crosses). Open symbols represent bubbles which fulfil the neon criterion, while crosses belong to those bubbles that fulfil additionally the oxygen temperature criterion.

\section{Application to the case of $\mathrm{BD}+30^{\circ} 3639$}

Our analysis of the X-ray emission from $\mathrm{BD}+30^{\circ} 3639$ 's bubble rests exclusively on the highresolution observations reported in Yu et al. (2009). We first used our ZP96-bubbles with WR composition to fix the characteristic plasma temperature $T_{\mathrm{X}}$. The result is seen in Fig. 2.

One sees that all bubbles nearly degenerate so that a unique plasma temperature can easily be derived from the oxygen and neon line ratios: $T_{\mathrm{X}}=1.9_{-0.2}^{+0.3} \mathrm{MK}$. This temperature value, obtained from the ZP96 bubbles, is also marginally consistent with magnesium. We emphasise that constant-temperature plasma models cannot provide a unique value for $T_{\mathrm{X}}$ (Fig. 2, right)!

For the further analysis, we have to check whether those bubbles from our grid that have the right temperature $T_{\mathrm{X}}$ can also reproduce the observed line ratios of carbon and neon with respect to oxygen. However, it turns out that none of our bubbles with homogeneous WR composition is able to reproduce the observed line ratios $\mathrm{Ne} / \mathrm{C}$ and $\mathrm{O} / \mathrm{C}$ (Fig. 3). The discrepancies amount to one order of magnitude, at least!

The large discrepancy between models and observation seen in the right line ratio diagram in Fig. 3 cannot be remedied by changing the input abundances of our WR composition because they are fixed by the analyses of the stellar wind of $\mathrm{BD}+30^{\circ} 3639$ (e.g. Marcolino et al. 2007), with the exception of neon whose abundance has been chosen by us. Instead, one has to conclude that the bubble of $\mathrm{BD}+30^{\circ} 3639$ contains, as the consequence of "evaporation", some hydrogenrich PN matter immediately behind the conduction front. The PN-matter contains much less carbon (and oxygen as well), so that even small amounts of PN matter will drastically change 

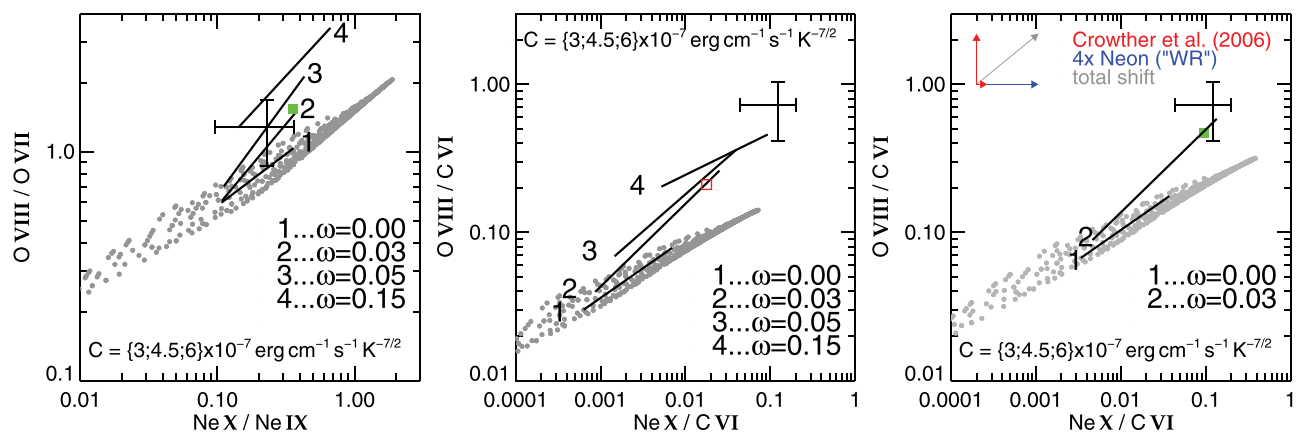

Figure 4. Left and middle: same as in Fig. 3 but with chemically inhomogeneous bubbles (black bars) added, labelled according to their $\omega$-values. The bars represent the mean positions of all bubbles with the same $\omega$. The square seen in all panels represent our "best-fit" model from the $\omega=0.03$ cloud (cf. Fig. 1). Right: positions of all models assuming a higher oxygen/carbon ratio as found by Crowther et al. (2006) and the neon/oxygen ratio increased by a factor of 2.5. The arrows indicate the shifts caused by the respective abundance changes.

the carbon line strengths, and to a lesser degree those of oxygen and neon lines because the corresponding ions reside in the inner, hotter bubble regions.

Figure 4 illustrates how the inclusion of PN matter changes the relevant line ratios. The discrepancy in the abundance sensitive panel (middle) is substantially reduced, although there is still some disagreement between the models and the observations. The square represents our "best-fit" model as a compromise between the temperature criteria and the abundance ratios: $\omega=0.03_{-0.01}^{+0.02}$ with age $=500 \mathrm{yr}$ (cf. Fig. 1 ), together with appropriate bubble size and X-ray luminosity.

So far we have implicitly assumed that the bubble's Ne abundance, which is not really constrained by photospheric/wind analyses, comes from the complete processing of CNO matter into $\mathrm{N}$, followed by conversion to Ne during a thermal pulse on the AGB. This leads to a mass fraction of 0.022 . By comparing the observed $\mathrm{Ne} / \mathrm{O}$ line ratio with the model predictions, we conclude that the $\mathrm{Ne}$ abundance in the bubble of $\mathrm{BD}+30^{\circ} 3639$ must be higher by a factor of about 2.5 (compared to oxygen), i.e. we have about equal amounts (by mass) of $\mathrm{O}$ and $\mathrm{Ne}$ (at least $\simeq 0.06)$ ! Combined with a higher $\mathrm{O} / \mathrm{C}$ ratio as proposed by Crowther et al. (2006), full consistency between both the temperature sensitive and abundance sensitive diagrams in Fig. 4 is achieved. In this case, the Ne content would be even higher (up to 0.09 by mass).

\section{Summary and conclusion}

We have developed a new tool for analysing the X-ray emission from wind-blown bubbles with thermal conduction. Application to existing high-resolution data for $\mathrm{BD}+30^{\circ} 3639$ shows that its X-ray line emission can only be explained if the bubble contains i) helium-, carbon-, and oxygen-enriched matter as found in the stellar photosphere/wind, especially enriched by neon, and ii) a shell of hydrogen-rich PN matter of about $3 \%$ by mass behind the conduction front.

It appears that heat conduction is a viable option for explaining the diffuse X-ray emission from wind-blown bubbles. More high-resolution X-ray observations are urgently needed for further testing our analytical bubble models. Of course, new analyses of existing low-resolution $\mathrm{X}$-ray spectra would also benefit from our heat-conduction chemically stratified bubble models.

\section{References}

Crowther, P. A., Morris, P. W., \& Smith, J. D. 2006, ApJ, 636, 1033

Dere, K. P., Landi, E., Young, P. R., et al. 2009, A\&A, 498, 915

Kastner, J. H., Montez, R.,Jr., Balick, B., et al. 2012, AJ, 144, 48

Marcolino, W. L. F., Hillier, D. J., de Araujo, F. X., \& Pereira, C. B. 2007, ApJ, 654, 1068 
Nordon, R., Behar, E., Soker, N., Kastner, J. H., \& Yu, Y. S. 2009, ApJ, 695, 834

Sandin, C., Steffen, M., Schönberner, D., \& Rühling, U. 2016, A\& A, 586, A57

Soker, N. 1994, AJ, 107, 276

Steffen, M., Schönberner, D., \& Warmuth, A. 2008, A\& $A, 489,173$

Toalá, J. A. \& Arthur, S. J. 2016, MNRAS, 463, 4438

Yu, Y. S., Nordon, R., Kastner, J. H., et al. 2009, ApJ, 690, 440

Zhekov, S. A. \& Perinotto, M. 1996, A\&A, 309, 648

Zhekov, S. A. \& Perinotto, M. 1998, A\&A, 334, 239

\section{Discussion}

De MARCO: Some born agains/[WR] have Ne mass fraction well above the value expected (few percent by mass). Is the Ne mass fraction were high enough to suspect a high mass progenitor? (higher than $\sim 2 \mathrm{M}_{\odot}$ )?

Schoenberner: No. The mass fraction of Ne in BD +303639 is $\sim 6 \%$ by mass and can be explained by evolution with overshoot in a $\sim 3 \mathrm{M}_{\odot}$ star.

TORRes-Peimbert: How important is the mixing of the envelope and the stellar wind at the conductive interface? Will it affect the overall nebula?

Schoenberner: Heat conduction across the bubble/nebula interface has an egligible influence on the nebular evolution. The evaporated mass is very small for the nebula, but important for the bubble to provide the observed X-ray luminosity, see Steffen et al. (2008, A\&A, 489, 173). 